Das Bleisalz besteht aus 40,34 Proc. oder 1 Atom Siuure und 59,66 Proc. oder 1 At. Oxyd, enthält kein Krystallwasser, löst sich in kaltem Wasser gar nicht, in siedendem sehr wenig und fällt daraus beim Erkalten in Blättchen wieder heraus, ist unlöslich in überschüssiger Säure und in Alkohol, fängt bei $120^{\circ} \mathrm{C}$. an gelb zu werden, schmilzt bei $135^{\circ}$ zu gelben Tropfen, die bei $140^{\circ}$ roth und bei $150^{\circ}$ zerselzt werden, giebt, in einer Röhre verkoblt, einen Rückstand, der, noch heifs in die Luft geschültet, glühende Funken mit nachziehendem weifsen Rauche bildet, entflammt sich bei Erhitzung mit Salpetersäure wie Pulver, entwickelt mit concentrirter Schwefelsäure anfangs einen dem Weinöl ähnlichen Geruch und später schweflige Säure.

Eisen wird unter Wasserstoff-Entwickelung von der Hydroxalsäure gelöst, und die Lösung hinterläfst eingedampft einen gummigen Rückstand.

Kupfer, und noch besser Kupferoxyd lösen sich in ihr, aber das bläuliche Salz krystallisirt nicht.

Quecksilberoxyd giebt ein weifses, fast unlösliches Salz, das Lackmus röthet.

Chromoxydul liefert ein sauer reagirendes Salz in durchsichtigen, farblosen, schiefbasigen Prismen.

\title{
IV. Ueber die Gummiarten;
}

von Hrn. R, T. Gućrin.

(Auszug aus den Ann. de chim. et de phys. T. XLIX p. 249.)

$\mathbf{U}$ nter dem Namen Gummi versteht der Verfasser alle die Pflanzenstoffe,' welche sich durch Salpetersäure in Schleimsäure umwandeln lassen, ohne dabei krystallisirbar zu seyn, wie der Milchzucker, dem sonst auch die nämliche Eigenschaft zukommt *). Hauptsächlich sind es

-) Um das Schwankende in der bisherigen Begriffsbestimmung vom Gurnmi fühlbar zu nachen, bemerkt der Verfasser, dafs Hr. Ro- 
drei Punkte, die er zu ermitteln beabsichtigt hat, nämlich: 1) Ob die ganz in Wasser löslichen Gummisorten einfache Pflanzenstoffe und unter einander identisch seyen. 2) $\mathrm{Ob}$ das in kaltem Wasser Lösliche der nur theilweis lösbaren Gummisorten auch Schleimsäure gebe und einerlei mit deın ganz löslichen Gummi sey. 3) Ob der unlösliche Theil der nur schwierig in Wasser löslichen Gummisorten ebenfalls Schleimsüure liefere und mit dem Bassorin zusanmenfalle. Das Resultat dieser Untersuchungen ist nun, dafs die in der Natur vorkommenden Gunmisorten drei verschiedene Gummistoffe enthalten, nämlich Arabin, Bassorin und Cerasin.

Das Arabin (den Namen hat Hr. Chevreul gebildet) macht den löslichen uud bei weitem grölsten Theil des arabischen Gummi's aus, findet sich aufserdem im Seneyalgummi und in Leinsaamenschleim. - Das Bassorin bildet die Hauptmasse des unlöslichen Theils vom Bassora- und vom Traganthgummi. - Das Cerasin endlich ist, neben Arabin, im Kirsch-, Aprikosen-, Pfirsichund Mandelgummi enthalten.

Eigenschaften des Arabins. Es ist durchscheinend, ohne Farbe, Geruch und Geschmack, im trocknen $\mathrm{Zu}$ stande zerreiblich und mit Glasbruch versehen, zwischen $150^{\circ}$ und $200^{\circ}$ C. weich und in Fäden zu zichen, vollkommen löslich in Wasser, (Haben 100 Theile Wasser 17,75 Arabin - Th. bei $20^{\circ} \mathrm{C}$., und 23,54 Th. bei $100^{\circ} \mathrm{C}$. aufgenommen, so ist die Lösung nicht mehr filtrirbar) unkrystallisirbar, nicht der Weingährung fähig, aber in Wasser gelöst an der Luft, nicht im Vacuo, sauer werdend, in wäfsriger Lösung durch Bleiessig fällbar, nicht durch kieselsaures Kali (gegen Thomson's Angabe).

biquet gerade das Gummi, welches Hr. Couverchel durch Einwirkung von Weinsäure aus Satzmehl bereitete, als Normalgummi betrachtet, weil es mit Salpetersäure keine Schleimsäure, sondern nur Kleesäure giebt. (Vergl. diese Annalen, Bd. XXII S. 426.) 
Mit Chlor giebt es, nach Hrn. G., keine Citronensäure, wie Hr. Vauquelin fand, sondern nach lüngerer Behandlung (z. B. zehnstündiger, einer Lösung von 1 Th. Arabin in $50 \mathrm{~Tb}$. Wasser) eine trübe Flüssigkeit, die mit der Zeit einen weifsen, flockigen Niederschlag absetzt, und nichts wie Salzsäure enthält (doch wobl auch unverändertes Arabin? $\boldsymbol{P}$.). Dieser Niedersclulag, den iubrigens Liebig nicht wahrnahm*), robthet, selbst nach

-) Siche Ann. Bd. XV S. 570. - Wie viel der Niederschlag betrug und ob alles Gummi darin verwandele worden war, wird'von Hrn. G. nicht gesagt. Sehr wahrscheinlich rührte der Niederschlag von einer beigemengten stickstoffhaltigen Substant. her.

Veranlalst durch dic obigen Angaben hat IIr. Simonin in Nancy folgende Versuche angestellt. Er löste 4 Unzen hellgelbes Senegalgummi in einem Liter Regenwasser auf und teitete innerhalb 24 Stunden 200 Liter Chlor hindurch. Die Flüssigkeit entfïbte sich allmälig und trübte sich dann schwach; nachdem sic eine Nacht über gestanden, hatte sie sich geklärt und eine Menge linienfürmiger Krystalle abgesetzt, dic sich indefs als Gyps erwiesen. Nach $\mathbf{A}$ bsonderung von diesen Krystallen wurde die Flüssigkeit ganz anf die frühere VVeise nochmals mit Chlor behandelt. Sie roch nun noch stärker wie zuvor nach Chlor und setzte abermals eine Menge Gypsnadeln ab, welche wahrscheinlich aus dem im Gummi enthaltenen Kalk und einem kleinen Schwefelsäuregebalt des nicht gewaschenen Chlorgases gebildet worden waren. Die mit Chlor behandelte Gunmilisung, welche eine grol'se Menge Chlorwasserstoflsüure enthielt, wurde mit Kreide gesättigt. Es entstand dadurch kein Niederschlag der auf Bildung von Citronensäure schliefsen liefs; allein die Flüssigkeit war weit weniger schleimig und das Gummi war daria fast ganz verschwunden. Zur Syrupsconsistenz abgeraucht und mit Alkohol vermischt, liefs sie eine pechartige Materic fallen, welche, nachdem sie lange mit $\Lambda$ lkohol gewaschen worden war, Feuchtigkeit anzog, und, in Wasser gelöst, auf Zusatz von Schwefelsäure viel Gyps fallen liefs, auch bei Erhitzung viele Chlorwasserstoffsäure ausgab. Da Hr. B raconnot glaubte diese Materie sey eine Verbindung von einer Pflanzensäure mit dehydrogenirtem Gummi, so wurde ihre wälsrige Lösung durch einen Ueberschufs von gepülvertem Kalk gesättigt und der entstandene Niederschlag durch verdünnte Schwefelsüure zersetzt. Dadurch gelang es denn wirklich eine pechartige Säure abzuscheiden, die 
dem Waschen unit kaltem Wasser, das Lackmus, schmekt stechend und hinterher bitter, riecht nach Chlor, und entbält, aufser diesem, Arabin und Stickstoff.

Mit Schwefelsäure behandelt, ganz auf die von Braconnot zur Verwandelung der Lumpen in Zucker angewandte Art, bekommt man, nach Hrn. G., eine alkoholische, syrupsartige, etwas saure Flüssigkeit; aus der

folgende Eigenschaften besafs. Sie war unkrystallisirbar, wenig löslich in Alkohol, zog Feuchtigkeit aus der Luft an, fallte essigsaures Bleioxyd, Kalkwasser, dieses in Ueberschufs genommen, und Barytwasser, brachte aber im! salpetersauren. Silber keine merbliche Verïnderung hervor. Mit kohlensaurcm Kalk gesättigt, gab sie ein sehr lösliches Salz, welches, getrocknet, gummiähnlich war, keine Feuchtigkeit anzog, und, in Wasser gelöst, von Schwefeisäure gefällt und zersetzt wurde.

Dieselbe Säure erhielt Hr. Simonin bei Behandlung des Zuckers mit Chlor, und er glaubt sie sey identisch mit der, welche man durch Salpetersäure aus dem Gunmi crueugen kann. (Ann. de chim. et de phys. T. L. p. 319.)

Gegen diese Notiz hat $\mathrm{Hr}$. Guérin später einige Einwendungen gemacht (Ann. de chim. et de phys. T. LI p. 222). Bei Wiederholung der Simonin'schen Versuche erhielt er nörnlich nicht ganz diè angegebenen Resultate. Als er die mit Chlor. behandelte Gummilösung durch überschïssigen Kalk sättigte, bekam er, unter Entwickelung eines starken Geruchs nach ReinetteAepfeln, cinen Niederschlag, und nachdem er diesen durch verdünnte Schwefelsïure zersetzt, die erhaltene Flüssigkeit zur $\mathbf{A b}$ scheidung des Gypses mit Alkohol vermischt, filtrirt und abigedampft hatte, einen liückstand, welcher gelb und unkrystallisirbar war, auf Lackmus sauer reagirte, kaum Feuchtigkeit. anzog, die Consistenz, von weichers Wachse hatte, in starkem Alkohol wenig löslich war, nach Einäscherung. Kalk hinterliefs, essigsaures Blei, salpetersaures Silber, Kalk- und Barytwasser fillte. Hieraus schliefst er, die von Simonin erhaltene Säure sey noch kalkhaltig gewesen, auch nicht identisch mit der durch Salpetersäure aus Gummi crzeugten; auch bernerkt er, es werde lange nicht olles Gumi durch das Chlor zersetzt, und der anfängliche Niederschlag rühre von einem Schwefelsäuregehalt des angewandten Chlors her. - Von einem stickstoffhaltigen weilsen Nicderschlag ist nicht weiter die Rede.

$\boldsymbol{p}$. 
süfse körnige Krystalle anschicfsen, die aber mit Bierhefe nicht gähren.

Salpetersäure, von 1,339 Dichte bei $10^{\circ} \mathrm{C}$., giebt nach ihrer Menge verschiedene Producte. Wird Arabin zu gleichen Theilen mit derselben erhitzt, greift sie dieses nur unvollkommen an. Nimmt man zwei Theile von ihr auf 1 Th. Arabin, so bekommt man aufser Schleimsäure eine andere Säure, die Sch cele für Aepfelsäure hielt *); nimmt man aber $400 \mathrm{Th}$. Salpetersäure auf $100 \mathrm{Th}$. Arabin, so bekommt man das Maximum von Schleimsäure, nämlich 16,88 Th. und aufscrdem wenig Klecsäure; grö. fserer Zusatz von Salpetersïure vermindert die Schleimund vermehrt die Kleesäure.

Eigenschaften des Bassorins. Es ist starr, unkrystallisirbar, halb durchscheinend, schwierig zu pülvèn, ohne Farbe, Geschmack und Geruch, unlöslich in kalten und warmen Wasser, blofs darin beträchtlich aufschwellend, auch unlösbar in Alkohol und nicht der Weingährung fihig; es gicbt mit Schwefelsüure eine krystallisirbare, süfse, abcr nicht der Weingährung fähige Substanz, und $100 \mathrm{Th}$. mit $1000 \mathrm{Th}$. Salpetersäure von 1,339 behandelt, liefern 22,61 Th. Schlcimsäure aufser Kleesäure.

Dargestellt wird das Bassorin, indem man BassoraGummi mit kaltem Wasser wäscht, so lange als dieses noch etwas aufnimmt, und dann den Rückstand erst auf einem Seihtuch und hernach in Wasserbade rom Wasser befreit. Das von Pelletier aus Gummiharzen abgeschiedene Bassorin ist viellcicht mit diesem einerlei.

Eigenschaften des Cerasins. Es ist starr, unkrystallisirbar, halb durchscheinend, leicht zu pülvern, obne Geschmack, Geruch und Farbe, unlöslich in Alkohol und nicht der Weingährung fähig. Es ist in kaltem Wasser unlöslich und schwellt blofs ein wenig darin auf; läfst man es aber längere Zeit, z. B. sechs Stunden mit Wasser sieden, so löst es sich auf und verwandelt sich dabei

-) Siehe den vorhergehenden Aufsatz. 
in Arabin; denn wenn man die Lösung zur Trockne abdampft, findet man, dafs der Rückstand sich nun in kaltem Wasser löst. $100 \mathrm{Th}$. Wasser lösen bei $20^{\circ} \mathrm{C}$. 13,15 Th., und bei $100^{\circ}$ C. 19,03 Th. ${ }^{*}$ ). Das Bassorin crleidet diese Umänderung durch das Sieden nicht. Da wun das Cerasin, wie Hr. G. gefunden, auch gleiche Elementarzusammensetzung wic das Arabin hat, so betrachtet er dasselbe als eine isomerische Modification des letzteren.

Dargestellt wird das Cerasin aus dem Kirschgummi, wie das Bassorin aus dem Bassoragummi, nämlich durch Waschen des Kirschgummi's mit kaltem Wasser so lange diefs noch etwas auszieht. Der Rückstand ist das Cerasin. Thomson belegt mit diesem Namen zwar auch den unlöslichen Theil des Traganlhgummi; diefs ist aber Bassorin, und uicht das Cerasin des Hrn. Guérin.

Elementar.Zusammensetzung der drei Gummistoffe. Nach Hrn. Guérin's Analysen ist dieselbe folgende:

Arabin und Cerasin **) Bassorin.

Gewicht, Atome. Gewicht. Atome.

\begin{tabular}{lrrrr} 
Kohlenstoff & 43,81 & 6 & 37,28 & 10 \\
Sauerstoff & 49,85 & 5 & 55,87 & 11 \\
Wasserstoff & 6,20 & 10 & 6,85 & 22 \\
Stickstoff & $\mathbf{0 , 1 4}$ & & & \\
\cline { 2 - 5 } & $\mathbf{1 0 0 , 0 0}$ & & $\mathbf{1 0 0 , 0 0 .}$ &
\end{tabular}

•) Gu ibort bemerkt hiegegen (.Journ. de chim méd. 1832, p. 432), dals sich $1 \mathrm{Th}$. Mimosengummi bei gewöhnlicher Temperatur schon in $2 \mathrm{Th}$. Wasser löse; vielleicht sind hier wie vorhin filtrirbare Lösungen gereeint.

$\boldsymbol{p}$.

-) Hr. Guérin giebt nicht die Resultate seiner Analyse des Cernsins, an; da er aber sagt, es habe gleiche Zusamruensetzung mit dem Arabiu, so ist es hier sogleich neben letzteres gestellt. Dagegen führt der Verfasser als nihere Bestandeheile des Cerasins an, vor dem Kochen mit Wasser: 90,6 Cerasin und 8,4 Wasser aufser 1,0 Asche, nach dem Kochen: 90,587 Arabin und 8,402 Wasser aufser 1,011 $\Lambda$ sche. Die nahe Gleichheit der Menge des Cerasins und Arabins in diesen beiden Fällen gebrauchı ex mit als Beweis der isomeren Unwandelung des ersten in das letzere 
Die geringe Menge des Stickstoffs im Arabin betrachtet der Verfasser als zufällig.

Nach diesen Gummistoffen zerfallen nun die verschiedenen Gummi-Arten in folgende drei Klassen.

- Ich mufs indels bekennen, nicht recht einzusehen, was IIr. Guérin hier beim Cerasin und in der Folge bei allen Educten aus den nur theilweis löslichen Gummiarten unter dem Wassergehalt eigentlich verstehe. Er trocknete alle diese Educte auf dern Wasserbade, also wohl bei $100^{\circ} \mathrm{C}$.; vor der Verbrennung scheint er diese Stoffe im Vacuo bei $125^{\circ} \mathrm{C}$. getrocknet zu haben. Der angegebene Wassergehalt wäre also der, welchen dic Substanzen bei $100^{\circ}$ einschliefsen und bei $125^{\circ}$ verlieren; bei $100^{\circ} \mathrm{C}$. enthält aber nacl Berzelius das Mimosengummi wenigstens kein Wasser, welches ihm durch Erhitzung mit Bleioxyd entzogen werden künute. Oder trocknete Hr. G. scine Educte auf dem Wasserbade nur in gewönlicher Temperatur, für dic auch offenbar der Vassergehalt der satürlichen Gummiarten gilt. Jedenfalls hätte dicls wohl gesagt zu werden verdient; allein die $A b-$ handlung des Verfassers enthält nicht das mindeste Detail sowohl in dieser Beziehung als überlaupt in Bezug auf die $\Lambda$ nalysen, wohl aber häufige Spuren einer nachlässigen Abfassung. So z. B. ist gewölnnlich neben der unmittelbaren Zusammensetzung aus Wasser und Arabin oder Bassorin oder Cerasin auch die entferntere aus Kohlenstoff, Wasserstoff und Sanerstoff angegeben, ohne dafs dabei bemerkt wird, dals sich die letztere, wie höchst wahrscheinlich, nicht auf das Ganze, sondern nur auf das in dem Gummi enthaltene bei $125^{\circ} \mathrm{C}$. getrockncte Arabin, Bassorin oder Cerasin beziehe. - Aus diesem Grunde sind die Resultate der Elementar-Analysen der zusammengesetzten Gurnmiarten hier übergangen, zumal sich diese, wenn die Verbältnisse der näheren Bestandtheile und dic Elementarbe'standtheile der cinfachen Gummistofle richtig angegeben sind, aus bciden berechnen liefsen. Uebrigens stimnit die für das Arabin gefundene Zusammensetzung weder mit Prout's noch mit Berzelius's Angaben. Prout's Resultatc (Annal. Bd. XII S. 270) geben

für das ungetrocknetc Mimosongummi die Formel $\mathrm{C}^{25} \dot{\mathrm{H}}^{30}$

für das bei $100^{\circ} \mathrm{C}$. getrocknete - - $\mathrm{C}^{25} \dot{\mathrm{H}}^{24}$

letzteres bukommt nach Berzclius - - $C^{26} \dot{\mathbf{H}}^{24}$ nach Guérin dagegen $\quad-\quad-C^{24} \dot{H}^{20}$ 
Erste Klasse; arabinhaltige.

Arabisches Gummi. Ist bald farblos, bald gelb, roth oder braun gefärbt, welche Farben es jedoch bei längerem Liegen im Sonnenschein, oder besser noch bei Erhitzung bis $100^{\circ}$ C. verliert. Befeuchtet, röthet es Lackmuspapier und schmeckt zuwcilen sauer. Es hat cine Dichte von 1,355. Seine wälsrige Lösung ist immer etwas trübe, und setzt auf dem Filtrum einc Substanz $\mathbf{a b}$, die bei Erhitzung Ammoniak ausgicbt, wic auch Saussure und Vauquelin fanden. Mclimals siedend mit starkem Alkohol behandelt, tritt es diesem Chlorcalcium, Chlorkalium, sauren äpfelsauren Kalk, essigsaures Kali, Chlorophyll und eine wachsartige Materie ab. Eingeäschert, hinterläfst es: kohlensaures Kali, kohlensauren Kalk, schr wenig phosphorsauren Kalk, Chlorkalium, Eisenoxyd, Thonerde, Kieselerde und Talkerdè.

Es besteht in 100 aus 79,4 Arabin und 17,6 Wasser aufser 3 Asche.

Senegalgummi bildet zuweilen faustgrofse, eiförmige, oft hohle Stücke von 1,436 Dichte, im Uebrigen aber denselben Eigenschaften wie das arabische Gummi. $100 \mathrm{Th}$. Wasser lösen bei $20^{\circ} \mathrm{C}$. 18,49 Th., bei $100^{\circ} \mathrm{C}$. 24,17 Th. Alkohol, Chlor, Schwefelsäure und Salpetersäure wirken auf dasselbe wie auf das arabische Gummi. 100 Th. geben, mit 500 Th. Salpetersïure behandelt, 16,7 Th. Schleimsäure aufser Oxalsäure.

Es besteht in 100 aus 81,1 Arabin und 16,1 Wasser aufser 2,8 Asche.

Leinsaamenschleim. Behandelt man ausgesuchten Leinsaamen bei ciner Temperatur von $50^{\circ}$ bis $60^{\circ} \mathrm{zu}$ mehren Malen eine halbe Stunde lang mit dem achtfachen Gewicht Wasser, seiht den Schlein ab und dampft ibn auf dem Wasserbade in einer Porcellanschale (in einer Metallschale würde er zu sehr adhäriren) zur Trockne, so bekommt man einc Masse, die röthlich, bröcklich, lcicht zu pülvern und unkrystallisirbar ist, eigenthümlich, ge- 
wissermafsen nach Osmazom riecht, Lackmus röthet, bei Erbitzung Ammoniak ausgiebt, sich nicht in Alkohol löst und in kaltem Wasser beträchtlich aufquillt.

Hiebei löst das kalte Wasser einen Theil, während ein anderer ungelöst bleibt. Die Lösung wird weder durch Galläpfelaufgufs noch durch Chlor gefällt, auch nicht durch Jod gebläut (nur dann, wenn man statt des ganzen Saamens Leinsaamenmehl genommen, weil diefs wahrscheinlich mit Kornmehl verfälscht ist). Hr. G. findet den ganzen Schleim bestehend aus 52,70 Proc. löslichen Theilen, 29,89 Proc. unlöslichen, und 10,30 Wasser aufser 7,11 Asche.

Das Lösliche hat im Ganzen die Eigenschaften des Arabins. $100 \mathrm{~Tb}$. Wasser lösen $18,01 \mathrm{Th}$. bei $20^{\circ}$, und 23,71 Th. bei $100^{\circ}$. Mit dem vierfachen Gewichte Salpetersäure erhitzt, giebt es 14,25 Schleimsäure aufser Klecsüure. Es besteht, nach Hrn. G. aus: 67,5 Arabin nebst etwas stickstoffhaltiger Substanz und 14,0 Wasser aufser 18,5 Asche *).

Das Unlösliche hat Hr. G. nicht näher untersucht.

$$
\text { Zweite Klasse; bassorinhaltige. }
$$

Bassoragummi bildet schwach gelbliche Stücke von mittlerer Grölse, die zuweilen bohl, zuweilen abgeplattet und gefurcht, manchmal auch mit Auswüchsen versehen sind und eine Dichte von 1,359 besitzen. An siedenden Alkohol tritt es Chlorophyll, eine wachsartige Substanz, essigsaures Kali, Chlorcalcium und sauren äpfelsauren Kalk ab. In kaltem Wasser quillt es stark auf, ein Theil löst sich, ein anderer bleibt ungelöst.

Der unlösliche Theil besteht aus Bassorin, nebst

-) Hr. G. giebt hier ebenfalls wieder die Elementarzusammensetzung, nämlich $\mathrm{C}^{8} \mathrm{O}^{5} \mathrm{H}^{9}$; wahrscheinlich bezicht sich auch diese nicht auf das gesamrote Lösliche des Leinsaamengummi's, sondern nur auf das darin enthaltene trockne Arabin. Auffallen mufs es aber immer, wie hier $1 \Lambda$ t. Wasserstoff zu wenig gefunden ist. $p$. 
phosphorsauren Kalk, Alaunerde, Kieselerde, Eisenoxyd und Magnesia.

Der lösliche Theil dagegen ist zusammengesetzt aus 81,2 Arabin und 12,3 Wasser aufser 6,5 Asche. $100 \mathrm{Th}$. Wasser lösen $17,28 \mathrm{Th}$. bei $20^{\circ}$, und $22,98 \mathrm{Th}$. bei $100^{\circ}$. - $100 \mathrm{Th}$. mit $400 \mathrm{Th}$. Salpetersäure erhitzt, geben 15,42 Th. Schleimsüure aufser Kleesäure.

Traganthgummi hat eine Dichte von 1,384; bis $40^{\circ}$ oder $50^{\circ}$ erhitzt läfst es sich leichter pülvern als in gewöhnlicher Temperatur; in Wasser schwillt es aufserordentlich auf, und wenn man den Schleim einige Wochen lang an der Luft stehen läfst, verbreitet es, besonders wenn die Temperatur etwas erhöbt ist, einen ähnlichen Geruch wie Stärkmehlkleister unter gleichen Umständen oder wie Buttersäure. Der durch Sieden aus dem Traganthgummi erhaltene Klcister wird von alkobolischer Jodlösung blau, herrührend von unlöslichem Stärkmehl (was übrigens bekannt ist), das nach Guérin's mikroskopischen Untersuchungen in kleinen Körnern die Traganthstücke bekleidet *). Kocht man Traganth mit dem 40fachen Gewicht Wassers und filtrirt die Flüssigkeit durch dreifaches Papier, so bläut das Durchgegangene Jodlüsung nicht, während das auf dem Filter zurückbleibende es thut.

Das Traganth besteht, nach Hrn. G., in 100 aus 53,3 Arabin, 33,1 Bassorin nebst unlöslichem Amylum und 11,1 Wasser aufser 2,5 Asche. Digerirt man 1 Th. Traganth lïngere Zeit kalt mit $100 \mathrm{Th}$. Wasser, filtrirt die Lösung, wäscht den Rückstand gehörig und dampft daś Gelöste im Wasserbade in einer Silberschale ein, so hat man dasselbe in zwei Theile zerlegt.

Das Lösliche hat die Eigenschaften des Arabins und

-) Diese Amylumkörnchen sind, nach Hrn. G., theils rund, theils länglich. Die runden weichen nur darin von den Anylumkörnchen der Kartoffeln ab, dafs ihr innerer Theil aus Arabin besteht, der der letztereu dagegen lösliches Amylum enthält. 
besteht aus 76,4 Arabin und 12,1 Wasser aufser 11,5 Asche. Hundert Wasser lösen $17,43 \mathrm{Th}$. bei $20^{\circ}$, und 23,34 Th. bei $100^{\circ}$. 100 Th. geben mit 400 Th. Salpetersäure 15,21 Schleimsäure aufser Kleesäure.

Das Unlösliche, das man auch durch hinreichendes Kneten des Traganth mit kaltem $W$ asser und nachheriges Trocknen in Wasserbade bereiten kann, ist schuppig, schmulzig weils, zicmlich leicht zu pülvern, unkrystallisirbar, ohne Geruch und Geschmack, unveränderlich an der Luft, unlöslich in kaltem und heifsem Wasser, nur sehr darin aufquellend und einen dicken Schleim bildend, unlöslich in Alkohol und blau werdend durch Jod. 100 Th. mit 1000 Th. Salpetersäure behandelt, geben 22,53 Tb. Schleimsäure aufscr Klecsäure. Es bestelit aus 77,02 Bassorin uebst unlöslichem Amylum, und 18,71 Wasser aufser 4,27 Asche.

\section{Dritte Klasse; cerasinhaltige.}

Kirschgummi hat eine Dichte von 1,475 und ist farblos oder gefürbt; röthet Lackmus und schneckt zuweilen sauer; schwillt im kalten Wasser langsam auf, ohne sich jemals ganz darin zu lösen, löst sich aber in siedendem Wasser bis auf einige Holzfaserchen vollständig und ist dann verïndert; wird von Alkohol aus der wälsrigen Lösung gefällt (gegen Thomson's Angabe); verbält sich gegen Licht, Wärne, Chlor, Alkohol, Schwefelsäure und Salpetersäure wic arabisches Gummi. 1000 Th. geben, mit $400 \mathrm{Th}$. Salpetersäure behandelt, 15,54 Th. Schleimsäure und überdiefs Oxalsäure. Es besteht aus 52,1 Arabin, 34,9 Cerasin und 12,0. Wasser anfser 1,0 Asche. Letztere enthält, mit Ausnahme des schwefelsauren Kali's, alle Bestandtheile der Asche des arabischen Gummi's.

Aprikosengummi, ganz dem Kirschgummi ähnlich und von 1,469 Dichte; giebt, mit dem vierfachen Gewicht Salpetersïiure erhitzt, 15,97 Proc. Schleimsäure und aufser- 
deın Oxalsäure; besteht aus 89,85 Arabin nebst Cerasin und 6,82 Wasser aufser 3,33 Asche.

Pflaumengummi, von 1,491 Dichte, sonst ganz dem vorhergehenden ähnlich, und unter gleichen Unständen wie jenes 15,78 Proc. Schleimsäure, aufser Oxalsäure, gebend; besteht aus 82,23 Arabin nebst Cerasin und 15,15 Wasser aufser 2,62 Asche.

Pfirsichgummi, Dichte 1,421, Eigenschaften wie beim vorhergehenden, neben Oxalsäure 14,99 Proc. Schleimsäure liefernd, und bestehend aus: 82,6 Arabin mebst Cerasin und 14,21 Wasser aufser 3,19 Asche.

Mandelgummi, Dichte: 1,453, Eigenschaften ganz wie alle vorhergehenden, und wie diese mit Salpetersäure behandelt, aufser Oxalsäure 15,03 Proc. Schleimsăure gebend. Zusammensetzung: 83,24 Arabin nebst Ccrasin und 13,79 Wasser aufser 2,97 Asche.

Zuletzt hat Hr. Guérin noch den Milchzucker untersucht, weil derselbe, seiner Meinung nach, aufser den Gummiarten ·der einzige Körper ist, welcher, mit Salpetersäure behandelt, Schleimsäure bildet*). Das Maximun von Schleimsäure, nümlich 28,62 Th., aufser Oxalsäure, erhält man, nach ihm, wenn 100 Th. Milchzucker mit 600 Th. Salpetersäure behandelt werilen, und man bekommt sie dabei in kleinen durch einander liegenden Krystallen, anscheinend rectangulären Prismen. $100 \mathrm{Th}$. Wasser lösen 10,91 Milchzucker bei $20^{\circ}$ C. und 96,7 Theile bei $100^{\circ}$. Der Milchzucker enthält 0,8 Procent Wasser und hinterlälst 0,02 Proc. Asche **).

-) Auch das Picromel giebt auf gleiche Art eine bedeutende Menge Schleimsäure.

$\boldsymbol{p}$.

-) Die nun folgende Untersuchung über die Frage: $O b$ die durch Salpetersäure aus den Gummiarten gebildete Aepfelsäure identisch sey mit der natürlichen, ist durch den vorhergehenden Aufsatz überflüssig gemacht.

$P$. 